\title{
Anabases
}

ANABASES Traditions et réceptions de l'Antiquité

$20 \mid 2014$

Varia

\section{Sur une étonnante conjonction}

Philippe Brunet

\section{OpenEdition}

Journals

Édition électronique

URL : http://journals.openedition.org/anabases/4886

DOI : 10.4000/anabases.4886

ISSN : 2256-9421

\section{Éditeur}

E.R.A.S.M.E.

\section{Édition imprimée}

Date de publication : 1 novembre 2014

Pagination : 71-77

ISSN : 1774-4296

\section{Référence électronique}

Philippe Brunet, «Sur une étonnante conjonction », Anabases [En ligne], 20 | 2014, mis en ligne le 01 novembre 2017, consulté le 21 octobre 2019. URL : http://journals.openedition.org/anabases/4886 ; DOI : 10.4000/anabases.4886 


\section{Sur une étonnante conjonction}

Philippe Brunet

Homère tRADUIT EN HEXAMĖTRES, tel devait être le titre de la rencontre de traducteurs et de chercheurs qui s'est tenue au Réfectoire des Cordeliers, le 26 mars 2012, avec le soutien du service culturel de l'université Paris-Sorbonne et de l'université de Rouen (ERIAC), lors du festival de théâtre antique des Dionysies, dont c'était la $7^{\mathrm{e}}$ édition.

Très vite le titre est devenu Homère en hexamètres, titre plus court privilégiant le mode rythmique, essentiel. Dire, entendre, composer ou traduire, toutes ces facettes de la même opération étaient implicites. Peu importe ce qu'on y faisait exactement, pourvu que ce fût en hexamètres !

Homère en était le prétexte. On allait donc entendre son épopée traduite en plusieurs langues. Mais on ne pourrait l'entendre que si tout avait été fait par les poètes pour qu'on fit sonner les six temps de l'hexamètre à voix haute, en battant la mesure.

Jean Mousset le fit un jour de 1530, d'après la légende :

Chante, Déesse, le cour furieux et l'Ire d'Achillès pernicieuse qui fut ${ }^{1} \ldots$

Voilà tout ce qui reste de sa traduction. Il fallut recommencer, plus de 450 ans après... dans des mots qui finalement ne furent pas très différents :

Chante, déesse, l'ire d'Achille Péléiade,

ire maudite qui fit...

Après avoir traduit et œuvré dans mon coin pendant plus de vingt ans, après avoir mis en chantier des laboratoires de scansion, des thiases homériques, des confréries de

1 Cité par A. D’Aubigné, lettre à Salomon Certon, CEuvres, Pléiade, Paris 1969, p. 823, cf. E. ETKIND, Un art en crise. Essai de poétique de la traduction poétique, L'Âge d'homme, Paris 1982, p. 134 et n.18. 
comédiens et de traducteurs, autour de la compagnie Démodocos, créée en 1995, alors que je revendiquais une forme de publication orale en performance pour ce travail sur la voix visant à réhabiliter l'approche expérimentale ${ }^{2}$, j’ai finalement publié sous la forme d'un livre ma traduction ${ }^{3}$. On pouvait désormais trouver en librairie une Iliade à scander, la partager à haute voix, l'enseigner à des étudiants, susciter des lecteurs au-delà du cercle des amis et de la troupe.

Un jour, à l'occasion d'un cours de master sur les traductions d'Homère en hexamètres, où il était question de Voss, Gneditch, glorieux prédécesseurs, et de nos expériences plus récentes, j’ai découvert que je n'avais pas été le seul ces dernières années à scander Homère dans ma langue : un américain, Rodney Merrill, venait de traduire l'Iliade, après l'Odyssée, dans la tradition de Longfellow, là où Richmond Lattimore était resté, de son propre aveu, un peu en deçà de l'hexamètre ${ }^{4}$ ! Mon collègue Miguel Olmos me faisait entendre des hexamètres castillans d'une Iliade d'Agustín García Calvo, polis par cinquante ans d'élaboration. Et en 2012, au moment où j'envisageais la tenue du colloque, je découvrais qu'un jeune philologue italien, Daniele Ventre, avait repris la tentative de Guarducci, Pascoli et Faggella, et s'apprêtait à publier une Iliade en hexamètres italiens.

Ce qui était nouveau, c'était que des langues conçues à tort ou à raison comme réfractaires à l'hexamètre devenaient le terrain convergent d'expérimentations que d'autres langues avaient naguère réussies : allemand de Johann Heinrich Voss (17511826), ou russe de Nikolaï Gneditch (1784-1833).

En France, après de précieuses expériences de vers mesuré à l'Antique au $\mathrm{XVI}^{\mathrm{e}}$ siècle, le phénomène s'était interrompu : l'échec plus large de la Renaissance, ou plutôt le coup d'arrêt brutal imposé à l'humanisme par les guerres de religion, puis plus tard la domination exclusive de formes littéraires promues par le pouvoir royal et l'Académie, rendirent inaudibles les premiers essais " métrifiés " des poètes et des musiciens. Malgré quelques tentatives résurgentes ici et là, on pouvait dire que la cause était plaidée, et définitivement perdue.

C'est seulement au $\mathrm{XX}^{\mathrm{e}}$ siècle, dans les années 1980, que quelque chose bouge. André Markowicz, traducteur du russe, amène le poète latin Catulle et des formes accentuelles gréco-russes pouchkiniennes à se rencontrer dans la langue française, avec un grand succès rythmique et bonheur d'expression ${ }^{5}$. Il faut que je rencontre André, un jour, en 1981, pour que je commence à envisager une démarche de traducteur à partir de ce que je scandais en grec ou en allemand. Il me fallait commencer à entendre en français la scansion d'Homère, de Pindare ou de Hölderlin. Ce qui n'était pas du tout évident.

2 Voir le dossier Iliade, publié après les lectures intégrales de la Sorbonne et d'Avignon en 2005.

3 Aux éditions du Seuil, en 2010.

4 R. LatTimore, The Iliad of Homer, Chicago 1951.

5 Le Livre de Catulle, L'Âge d'Homme, Paris 1985. Travail annoncé dans l'ouvrage d'E. ETKind, Un art en crise, op. cit. 
De même, pour apprendre à scander, il faut qu'un jour quelqu'un vous l'apprenne. Avant on ne sait pas. Après on sait et on peut progresser. P. Fortassier, professeur au Lycée Louis-le-Grand, m'a appris à battre la mesure en lisant Virgile. S.G. Daitz, professeur à l'université The City of New York, m'a ouvert l'oreille au chant des voyelles grecques. Grâce à André, je me suis mis à guetter, à discipliner les accents du français. À rythmiser les groupes de syllabes, à traquer l'accent complémentaire, à faire entrer les noms propres dans l'hexamètre. À dire, puis à faire dire ces vers à des jeunes gens, étudiants, comédiens, qui n'avaient pas d'a priori. Ce fut le début du Théâtre Démodocos.

Ainsi, à la fin du $\mathrm{Xx}^{\mathrm{e}}$ siècle, sur les décombres d'un vers syllabique français qui n'en finissait pas de renaître et de mourir, la recherche d'une vocalité (sensible dans les mouvements de poésie sonore, ou dans d'autres, dits actuels, comme le rap ou le slam) tendait à réhabiliter le rythme, l'accent, le ton, et les premières expériences de rythme à l'Antique. De Catulle à Homère, ce qui se jouait, c'était autant un retour à une tradition antique perdue que l'inscription de la voix dans l'écriture. Le rythme égal, dactylique, battu dans les six mesures du vers, s'est fait épopée, mise en voix de la parole.

Revenir à Homère n'était pas anodin. Par delà les discours archéologiques ou comparatistes, et les disputes académiques, où se rêve la quête d'une origine impossible à déterminer, Homère et l'épopée en général représentaient le lieu par excellence de la profération rythmée, de la parole éprouvée comme écoute et comme partage. Il devenait donc opportun de réunir ces récents traducteurs, diseurs, ou leurs témoins et historiens : tous devraient, d'une manière ou d'une autre, témoigner sur l'expérience homérique.

Agustín García Calvo, malgré son état de santé très fragile, nous fit l'honneur de venir de Madrid rassembler encore une fois l'expérience du Maitre et le savoir du professeur ; à cet art, il ajouta celui de faire entendre les hexamètres enregistrés par lui ${ }^{6}$. Sa soudaine disparition en 2012 ne fit qu'accroître à nos yeux le prix de cette ultime conférence parisienne. L'intervention du Maître a répondu à notre attente : car tous, plus ou moins, nous portons témoignage de cette difficulté à faire coexister dans nos vies la philologie et l'art, la science et la poésie. Or, la science procède de l'art aussi inéluctablement qu'Homère précède Aristote. C'est par l'expérience de la scansion à voix haute, et par l'expérience de la traduction, que Maître Garcia Calvo a poursuivie pendant plus de cinquante années, que s'est développée une vision de l'objet philologique, moins idéalisée, plus pertinente, qui vient salutairement à la rencontre de nos diverses positions.

Rodney Merrill, traducteur nomade de l'Odyssée avant que de l'Iliade ${ }^{7}$, nous apporte le concours de la langue anglaise ramenée à la battue de la scansion homérique. Une vraie gageure, qui, cinquante ans après la traduction rythmée, mais non métrique,

6 Homero. Iliada, versión rítmica y prolegómenos de Agustín García Calvo, Zamora, Lucina 1995. 7 R. Merrill (trad.), The Odyssey of Homer, Ann Arbor 2002 ; The Iliad of Homer, Ann Arbor 2007. 
de R. Lattimore ${ }^{8}$, va au-delà des nombreux essais qui avaient été donnés en langue anglaise sans que jamais quelqu'un allât jusqu'au bout.

Sur le versant roman de l'Europe, Daniele Ventre vient apporter des lumières sur son hexamètre et faire sonner son texte de l'Iliade tout fraîchement paru ${ }^{9}$. Après les hexamètres de Manlio Faggella, que les Italiens n'avaient jamais vraiment voulu accueillir mais qui gagneraient à être relus aujourd'hui ${ }^{10}$, il apporte l'évidence de nouveaux hexamètres. Souhaitons-lui plus de chance au pays de Vicenzo Monti ! À l'heure où paraîtra le dossier Homère en hexamètres, son Odyssée, imminente, sera sans doute à son tour parue ${ }^{11}$.

Après les quatre plaidoyers des traducteurs d'Homère, du côté des pays baltes, Janika Päll, helléniste, vient témoigner sur les tentatives d'établir un hexamètre dans une langue quantitative, l'estonien, qui possède l'opposition entre la brève et la longue, et même un troisième degré pertinent, la super-longue !

En écho au travail de D. Ventre, Cristina Noacco, médiéviste italienne à l'université Jean Jaurès de Toulouse, se fait historienne pour retracer les origines de ce vers dans la démarche de Giovanni Pascoli.

Smaranda Badilita vient apporter son témoignage sur un cas de coexistence, dans la langue roumaine, de deux traductions de l'Iliade, celle de George Murnu (18681957) et celle plus récente de Dan Sluşanschi (1943-2008), qui œuvrèrent tous deux en voisins de l'archipel grec, habitants d'une même communauté balkanique.

À ces langues vient s'ajouter le témoignage d'un traducteur brésilien, Rodrigo Tadeu Gonçalves : il ouvre le dossier au continent sud-américain et nous fait découvrir, parmi d'autres voix brésiliennes, celle du poète Carlos Alberto Nunes, ainsi que l'activité de l'université de Parama, qui encourage la traduction poétique comme activité universitaire.

En France, à la parution de mon Iliade aux éditions du Seuil en 2010, de nombreux comptes rendus ont paru dans la presse ; un groupe d'écrivains méditerranéens réunis par Pierre Assouline (prix Écrimed) m'a remis un prix, ainsi que l'Académie française. La plupart des critiques se sont montrés sensibles au travail rythmique, à la dimension vocale dont la traduction porte la marque, mais en réalité rares sont ceux qui se prononcèrent sur le fond. Le débat s'est poursuivi aux Assises d'Arles en 2011 avec André Markowicz. Michel Volkovitch, présent lui aussi en Arles, nous a dit contradictoirement son soutien et son incompréhension ${ }^{12}$.

8 R. Lattimore, The Iliad of Homer, Chicago 1951.

9 D. Ventre (trad.), Omero, Iliade, préf. L. Spina, éd. Mesogea, Messina 2010.

10 Iliade, Bari, 1923, Odyssea, Bari 1925.

11 L'Odissea vient de paraître aux éditions Mesogea en 2014.

12 Lors de l'atelier, et plus tard : M. Volкоviтch, «Un saint chez Homère ", Pages d'écriture $\mathrm{n}^{\circ} 116$, en mai 2013, http://www.volkovitch.com/rub_carnet.asp?a=pe116. 
Il m'a donc semblé utile ici de formaliser un peu plus les données prosodiques et métriques à l'intention des futurs auteurs de traités, jusqu'ici à peu près unanimement sourds à ces expériences.

Notable exception dans l'indifférence générale à la poésie française accentuelle, Juliette Lormier apporte le concours de sa recherche de doctorante lilloise et réalise ainsi une première percée dans ce domaine encore limité à quelques expériences poétiques et théâtrales. Par un puissant raccourci, je me retrouve associé, dans son article, à Jean-Antoine Baïf, helléniste, poète et fin rythmicien qui collabora avec les musiciens Claude Le Jeune et Jacques Mauduit, à une époque que les antagonismes religieux allaient bientôt faire oublier.

Cette rencontre est centrée sur Homère, mais elle porte en germe ses prolongements. Voilà pourquoi ce travail issu de la traduction de Catulle revient à la poésie latine après un détour par Homère. Aymeric Münch et Guillaume Boussard, co-traducteurs de L'Orestie d'Eschyle de 2010 pour le Théâtre Démodocos, présentent leurs travaux en cours. G. Boussard justifie ses choix pour sa version du poème de Lucrèce, qu'il récite à deux voix à ses auditeurs, en symbiose avec des pièces pianistiques interprétées par E. Lascoux ; A. Münch apporte l'aboutissement de presque dix ans de travail consacrés à traduire les Géorgiques de Virgile depuis qu'en 2000 nous jouâmes sa version de l'épisode d'Orphée ${ }^{13}$.

E. Lascoux, musicien et helléniste, passionné de rythmos grec, évoque la voix homérique, scandée directement dans le grec ou " transcandée ", plutôt que traduite, dans nos différentes langues. François Cam présente la mélodie qu'il composa naguère sur ma traduction du préambule de l'Odyssée : Muse, dis-moi... Par la suite, il nous arriva de chanter cette mélodie sur le texte grec lui-même, par un parcours inverse très étonnant qui nous fit remonter du français au grec ancien.

Le texte que je présente ici a été en partie écrit pour servir à Gilles de Rosny : auteur d'un programme de scansion de l'hexamètre homérique grec, ne pouvait-il pas tenter de scander automatiquement les hexamètres français ? C'est chose faite, ou presque. Gilles de Rosny a mis son savoir au service de la poétique d'Homère pour la deuxième fois. Il nous présente les deux logiciels ${ }^{14}$.

Il manquerait ici Jesper Svenbrö ${ }^{15}$, qui m’avait naguère alerté sur les récupérations que les nazis, en Suède, avaient faites des traductions en hexamètres. Est-ce pour cette raison que l'acteur Bruno Ganz, dans un enregistrement, témoigne, après la guerre, d'un refus de toute métrique quand il lit les poèmes allemands de Hölderlin ? C'est vrai

13 J'ai mis en scène Orphée pour le Thêâtre Démodocos en 2000, et L'Orestie en 2010-13 après une première tentative en 1997-98.

14 Programme Scande\&Chante en ligne sur www.homeros.fr ; programme Scande\&Marque au stade expérimental.

15 L'auteur de Phrasikleia, anthropologie de la lecture en Grèce ancienne, Paris 1988. 
que le terrain des études anciennes est miné. Mais la métrique, pas plus que l'architecture grecque, n'appartient au fascisme.

Voilà pourquoi la dimension politique n'est pas absente de notre démarche. Cette approche esthétique sera d'autant plus soutenue et digne d'exister qu'elle est absolument éloignée de toutes tentations néo-nazies, que celles-ci relèvent du fantasme indoaryen ou du pensoir heidegerrien. Elle va dans le sens de croisements entre les langues et les cultures, et non dans la valorisation d'une pseudo-identité enracinée dans une prétendue ontologie, fût-elle celle de l'art ou de la langue. On ne peut laisser Homère, Héraclite ou Hölderlin aux nationaux-socialistes ou à leurs épigones. La scansion pied à pied n'est pas la mise au pas : elle est alternance, respiration, rythme, liberté d'expression dans le chant de la parole. Elle n'est pas retour à une quelconque origine, ni souci ni authenticité, ni même institution d'une quelconque académie.

Je salue M. Yann Migoubert, complice d'autres Batrachomyomachies, et directeur du Service Culturel de l'université Paris-Sorbonne, qui a spontanément accepté et soutenu la programmation de la rencontre pendant nos Dionysies ; M. Miguel Olmos, directeur du laboratoire ERIAC de l'université de Rouen, qui a facilité la venue de M. Agustín García Calvo et assuré l'édition de sa conférence en accord avec Mme Isabel Escudero ${ }^{16}$; Mme Roseline Bigot, qui a généreusement ouvert le Réfectoire des Cordeliers au festival des Dionysies 2012 pour la sixième année consécutive ; MM. Manuel Mayer et Éric Pelladeau, régisseurs des Dionysies, qui ont contribué à la qualité sonore et visuelle de la journée.

$\mathrm{Si}$ je bouclais là ce dossier, il aurait manqué une voix ; en son absence, la synthèse risquait de tourner à la récupération. Quelle que soit l'objectivité des justifications faites a posteriori, par moi, ou par tel ou tel traducteur, quelles que soient la valeur historique des précédents, l'évidence des expériences parallèles et la réalité de nos expériences aédiques, il restait ce fait, insolite, simple et têtu effectivement : un passeur de Pouchkine avait traduit Catulle, parlant russe en français, ou retrouvant Pouchkine en Catulle. Et sans lui, rien n'aurait été. Ce ne sont jamais des schémas qui se transmettent. Avant les systèmes, il y a les gens, les individus, leur histoire ; avant la métrique, la voix. C'est irréductible, comme la voix d'André Markowicz. Ou comme tout ce que cette voix supposait, emmenait avec elle.

André m'adresse le texte qui suit, par lequel les jours déjà lointains de l'école buissonnière redeviennent tout à coup proches et palpables.

Philippe BRUNET

Université de Rouen (ERIAC)

Faculté des lettres et sciences humaines

76821 Mont-Saint-Aignan cedex

demodocos@demodocos.com

16 La transcription de la conférence fut réalisée par Ricardo Dorado et Ana Leal. 
Les principales traductions d'Homère qui font l'objet de ce dossier :

A. Annist, K. Reitav, Homère, L'Iliade, Eesti Riiklik Kirjastus, Tallinn 1960 [estonien].

J. Bergmann, Homère, L'Odyssée IX, 1889, EÜS, Album 1, p.102-118 [estonien].

Ph. Brunet, Homère, L'Iliade, Seuil, Paris 2010, Point Seuil, Paris 2012 [français].

A. García Calvo, Homero. Iliada, versión rítmica y prolegómenos de..., éd. Zamora, Lucina 1995 [espagnol].

R. Merrill, The Odyssey of Homer, Ann Arbor 2002; The Iliad of Homer, Ann Arbor 2007 [anglais].

G. Murnu (1868-1957), Homer, Iliada, Bucarest 1912 [roumain].

C. A. Nunes, Homero, Ilíada, 1a ed. Atena, São Paulo 1945 [portugais du Brésil].

C. A. Nunes, Homero, Odisséia, tradução C. A. Nunes, ed. Melhoramentos, São Paulo 1962 [portugais du Brésil].

A. ÖPIK, Homère, L'Odyssée I-XII, Varrak, Tartu 1938 [estonien].

G. Pascoli, Poesie e prose scelte, éd. C. Garboli, Mondadori, Milan 2002 [italien].

D. Slusanschi (1943-2008), Homer, Iliada, traducere în hexametri, cu o postfață, o bibliografie esențială şi indici de..., Paideia, Bucarest, 1998 [roumain].

D. Ventre, Omero, Iliade, préface de L. Spina, éd. Mesogea, Messina 2010 [italien].

\section{Autres traductions}

J.-A. DE BAÏF, Etrénes de poézie fransoeze an vers mezurés, Paris 1574, incluant les Besognes et les Jours d'Hésiode [français].

A. Markowicz, Le Livre de Catulle, L'Âge d'Homme, Paris 1985 [français].

\section{Traductions françaises en cours}

G. Boussard, Lucrèce, La Nature des choses.

Ph. BRUnet, Homère, L'Odyssée.

A. MüNCH, Virgile, Géorgiques. 